\title{
Comparative Analysis of MSER and DTW for Offline Signature Recognition
}

\author{
Mohammad Basil, Bharti Gawali \\ Department of computer science and information technology, \\ Dr. Babasaheb Ambedkar Marathwada University, Aurangabad (M.S), \\ Aurangabad Maharashtra India
}

\begin{abstract}
This paper describes the signature as a one of the most robust and widely used trait in biometric verification. The signature is also known as behavioral biometric property. We present comparative analysis of offline signature recognition based on Dynamic Time Warping (DTW) and Maximally Stable Extremely Regions (MSER). The MSER system reported accuracy of $95.371 \%$ where FAR is $5.25 \%$ and FRR is $4 \%$ whereas DTW system reported accuracy of $70.5 \%$ and average horizontal projection WER $70 \%$ and average vertical projection WER of $71 \%$.
\end{abstract}

\section{Keywords}

Signature, Dynamic Time Warping (DTW), Maximally Stable Extremely Region (MSER), FAR, FRR, TAR, TRR.

\section{INTRODUCTION}

The current area is an authentication which is dependent on Signature. It is one of the crucial way of authenticating individuals in various domain namely banks institutions, industries, and safeguarding documents. It have played a vital role over years; more than 30 years but still it have not reached its fullest (state-of-art) . [1,2].

Signature recognition is a behavioral biometric which refers to methods for uniquely recognize humans, based on one or more intrinsic physical or behavioral traits. It can be differentiated in two ways: static (offline) and dynamic (online) signature recognition. In static signature recognition the signature database is collected on a piece of paper, digitized with the help of scanner, the shape and size are the basic parameters used for static signature recognition, and in dynamic signature recognition; signatures are collected differently with the help of signature pad (device), it records the motion of the stylus while the signature is produced, and includes location, and possibly velocity, acceleration and pen pressure, as functions of time $[1,2,3]$

In the biometric era, signature verification is a robust and has dynamic scope. The signature verification means recognizing the signature on the basis of specific features also it gives the response of authentication.

A robust system has to be designed in a way it should not only be able to consider these factors but also detect various types of forgeries $[4,5]$.

The rest of this paper is organized as follows: section 2 the database available for signature recognition.Section 3 describes the techniques: Maximally Stable Extremely Region (MSER ) and Dynamic Time Warping (DTW) in details. The experiment analysis is explained in section 4. Performance, Results \& conclusion and reference are in section 5 and 6 respectively.

\section{DATABASE DESIGN}

This research contributed towards the creation of offline signature database. The signatures are collected using either black or blue ink, on a white A4 sheet of a paper, with forty signatures per page from thirty four volunteer. Using MF4350D scanner the scanner the four signatures are digitized, with 300-dpi resolution in 256 grey levels. The volunteer selected are from students of Department of Computer science and Information Technology, Dr. Babasaheb Ambedkar Marathwada University. The 16 subject are from 20-25 age group and 18 subjects are in the 25-30 age group. The total size of dataset is 1360 . The signatures are stored in the offline database is scanned image files in jpg format with standard size of $200 \times 100$ pixels. The basic preprocessing operations include the spatial alignment of the image, followed by binarization, which converts the image files into logical matrices and finally a skeletonization process that extracts the thin path or contour of the signature

\section{METHODOLOGY}

We present the description of the two techniques: MSER (Maximally Stable Extremely Region) and DTW (Dynamic Time Warping)

\subsection{Maximally Stable Extremely Regions (MSER)}

The choice of a powerful set of feature is crucial in signature recognition systems. The features used must be suitable for the application and for the applied classifier. In this system, maximally Stable Extremely Region (MSER) features are used such as grid features and global features. The global features provide information about specific cases concerning the structure of the signature grid feature. This method of extracting a comprehensive number of corresponding image elements contributes to the wide-baseline matching, and it has led to better stereo matching and object recognition algorithms. The original algorithm is proposed by Mates [6] is in the number of pixels. It proceeds by first sorting the pixels by intensity. This would take time, using BINSORT. After sorting, pixels are marked in the image, and the list of growing and merging connected components and their areas is maintained using the union-find algorithm. This would take time. In practice these steps are very fast. During this process, the area of each connected component as a function of intensity is stored producing a data structure. A merge of two components is viewed as termination of existence of the smaller component and an insertion of all pixels of the smaller component into the larger one. In the extremely regions, the 'maximally stable' ones are those corresponding to thresholds where the relative area change as a function of relative change of threshold is at a local minimum, i.e. the Maximally Stable Extremely Regions ( MSER) are the parts of the image where local binarization is stable over a large range of thresholds[7][8]. 
The component tree is the set of all connected components of the thresholds of the image, ordered by inclusion. Efficient (quasi-linear whatever the range of the weights) algorithms for computing it do exist [9]. Thus this structure offers an easy way for implementing MSER [10]. The steps for Maximally Stable Extremely Regions (MSER) feature extraction are as follows [11].

Sweep threshold of intensity from black to white, performing a simple luminance thresholding of the image.

$>$ Extraction of the connected components ("External Regions").

$>$ Find a threshold when an extremely region is "Maximally Stable", i.e. local minimum of the relative growth of its square.

The border (corner) point of the signature play an important role for the identification of signature match area, the figure 1 (A) described the corner point detection of the signature. The Extracted Maximally Stable Extremely Regions (MSER)feature of signature is described in figure1 (B).

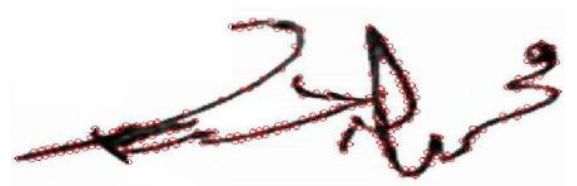

A

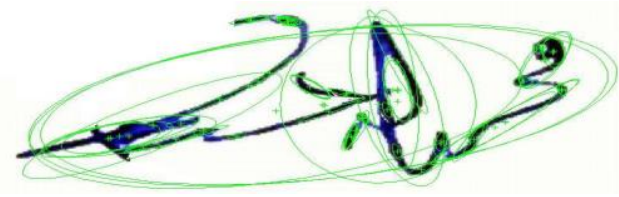

B

Figure (A) The corner point detection of signature recognition (B) Extracted feature of signature using Maximally Stable Extremely Regions ( MSER )

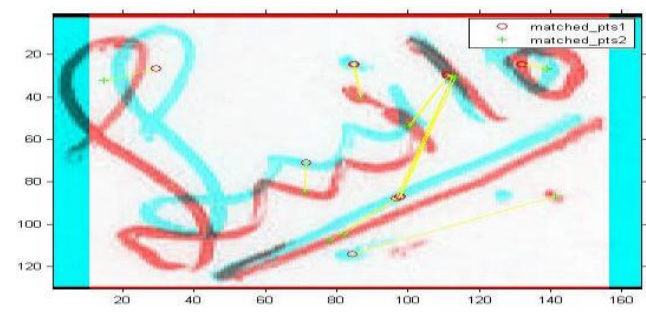

(A)

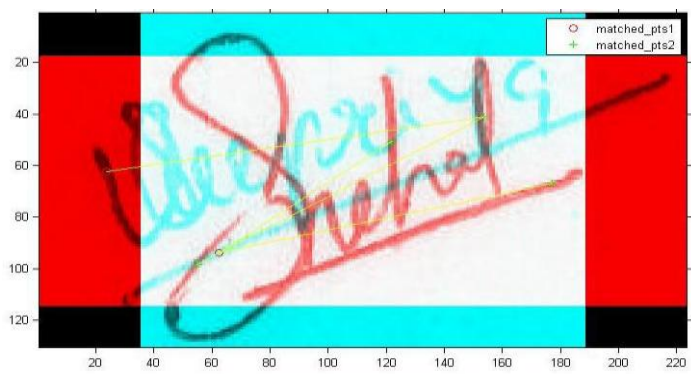

( B)

Figure 2. The graphical representation of matching of two [A] same signature [B] Different signatures

\subsection{Dynamic Time Warping (DTW)}

It is well known in its classification persons based on the similarity matching of time series obtained from signatures. The most advantage of it is that it is capability of matching two time series $\mathrm{Q}=(\mathrm{q} 1, \mathrm{q} 2 \ldots . \mathrm{qm})$ and $\mathrm{T}=(\mathrm{t} 1, \mathrm{t} 2, \ldots \ldots \mathrm{tn})$ of unequal length $\mathrm{n} \neq \mathrm{m}$.

The classification is also based on its results to a high performance even for time series of small length. It basically measures the similarity of the two time series $(\mathrm{Q}, \mathrm{T})$ in terms of the distance between them after they have been warped together. The distance value is found by minimizing a cumulative cost which is defined by the Euclidean distances between all matches ( $\mathrm{i} \mathrm{j} \mathrm{q,} \mathrm{t} \mathrm{).} \mathrm{Given} \mathrm{an} \mathrm{unknown} \mathrm{sample} Q$, The Dynamic Time Warping (DTW) based classifier calculates the distances to all references $\mathrm{T}$ among a population (persons, characters, etc.), sorts them by distance, and returns a list of nearest references. The minimum-distance of the top best match decides for classification $[12,13,14,15]$.

\section{EXPERMINTAL ANALYSIS}

The signatures were cropped in the image box of $200 \times 100$ pixels. All the images are cropped and converted into the binary images. The following features are from the horizontal and vertical (histogram) projections collected from the signatures both for training and testing based on the border clearing. The background is white colour and the signatures dark hence with this signature is allocated in the image more easily through its border lines.

The Dynamic Time Warping (DTW) algorithm is based on the dynamic programming of finding an optimal match between two sequences of feature vector. The dissimilarity between reference and the test node is represented by $\mathrm{D}(\mathrm{M}, \mathrm{N})$.

$\mathrm{D}(\mathrm{m}, \mathrm{n})=\mathrm{d}(\mathrm{m}, \mathrm{n})+\min [\mathrm{D}(\mathrm{m}-1, \mathrm{n}), \mathrm{D}(\mathrm{m}, \mathrm{n}-1)]$

DTW dist=D(M,N)

The training of system using Dynamic Time Warping (DTW) is performed by equation (2) where G1 is the training score.

$\mathrm{G} 1=\frac{2}{d(d-1)} \sum_{\mathrm{i}-1}^{\mathrm{d}-1} \sum_{\mathrm{j}-\mathrm{i}+1}^{\mathrm{d}} \operatorname{DTW} \operatorname{dist}(\mathrm{G} 1, \mathrm{Gj})$ : where $\mathrm{i}, \mathrm{j} \leq \mathrm{d}[2]$

The verification of the system using Dynamic Time Warping (DTW) is performed by equation (3) where G2 is verification score.

$$
G 2=\frac{1}{d} \sum_{i-1}^{d} D T W \operatorname{dist}(G t, G i: \text { where } i \leq d
$$

The decision to accept or reject signature is based on the value of the threshold score given by equation

Score $=$ G1/ G2 [16]

The detail of minimum-distance of Horizontal projection and Vertical projection in the classification are discussed in table 1. 
Table 1.The detail of minimum-distance of Horizontal projection and Vertical projection classification

\begin{tabular}{|l|l|l|l|}
\hline S.No. & Signatures & $\begin{array}{l}\text { Horizontal } \\
\text { projection }\end{array}$ & $\begin{array}{l}\text { Vertical } \\
\text { projection }\end{array}$ \\
\hline 1. & Signature 1 & 0.010 & 0.033 \\
\hline 2. & Signature 2 & 0.016 & 0.026 \\
\hline 3. & Signature 3 & 0.01 & 0.028 \\
\hline 4. & Signature 4 & 0.08 & 0.100 \\
\hline 5. & Signature 5 & 0.08 & 0.026 \\
\hline 6. & Signature 6 & 0.011 & 0.100 \\
\hline 7. & Signature 7 & 0.10 & 0.034 \\
\hline 8. & Signature 8 & 0.08 & 0.033 \\
\hline 9. & Signature 9 & 0.08 & 0.042 \\
\hline 10. & Signature 10 & 0.010 & 0.041 \\
\hline
\end{tabular}

The Maximally Stable Extremely Regions ( MSER) feature is the combination of the centroid, Axes location, orientation as well as pixel list point, Detail of Maximally Stable Extremely Regions ( MSER) feature set are described in table 2.

Table 2. The Extracted Maximally Stable Extremely Regions MSER Feature of signature

\begin{tabular}{|l|l|l|l|l|}
\hline $\begin{array}{l}\text { Match } \\
\text { point }\end{array}$ & \multicolumn{2}{|l|}{ Location } & Scale & $\begin{array}{l}\text { Orientati } \\
\text { on }\end{array}$ \\
\hline & Min & Max & & \\
\hline 1 & 190.94 & 243.29 & 1.600 & 1.7280 \\
\hline 2 & 169.80 & 229.51 & 8.2667 & 1.8837 \\
\hline 3 & 1.8540 & 258.73 & 2.1333 & 2.0377 \\
\hline 4 & 163.77 & 292.35 & 1.6000 & 2.2402 \\
\hline 5 & 163.77 & 292.35 & 1.6000 & 2.2402 \\
\hline 6 & 153.66 & 303.32 & 1.8867 & 2.3237 \\
\hline 7 & 153.66 & 303.32 & 1.8867 & 2.3237 \\
\hline 8 & 292.52 & 274.05 & 1.600 & 1.7948 \\
\hline 9 & 200.01 & 355.60 & 2 & 1.9420 \\
\hline 10 & 200.01 & 355.60 & 2 & 1.9420 \\
\hline
\end{tabular}

Table 3. False acceptance rate and false rejection rate for the Maximally Stable Extremely Regions ( MSER ) system

\begin{tabular}{|l|l|l|l|l|}
\hline $\begin{array}{l}\text { Test } \\
\text { signature }\end{array}$ & $\begin{array}{l}\text { No. of } \\
\text { token } \\
\text { passed }\end{array}$ & FRR (\%) & FAR $(\%)$ & Accuracy \\
\hline Subject 1 & 40 & 5 & 7.5 & 87.5 \\
\hline Subject 2 & 40 & 7.5 & 0 & 80 \\
\hline Subject 3 & 40 & 2.5 & 10 & 87.5 \\
\hline Subject 4 & 40 & 5 & 2.5 & 90 \\
\hline Subject 5 & 40 & 5 & 7.5 & 87.5 \\
\hline Subject 6 & 40 & 2.5 & 7.5 & 90 \\
\hline Subject 7 & 40 & 7.5 & 10 & 82.5 \\
\hline Subject 8 & 40 & 2.5 & 2.5 & 95 \\
\hline Subject 9 & 40 & 2.5 & 10 & 92.5 \\
\hline Subject 10 & 40 & 0 & 5 & 95 \\
\hline Average & 40 & 4 & 5.25 & 95.374 \\
\hline
\end{tabular}

\section{5 .PERFORMANCE AND RESULTS}

\subsection{Performance Parameters for Maximally} Stable Extremely Regions (MSER)

Performance Analysis of the system includes an evaluation of all possible errors - False Acceptance and False Rejection give a fairly good idea of the efficiency for verification. The True Acceptance Rate (TAR) and the True Rejection Rate (TRR) are the correct-classification rates.

\section{a) False Acceptance Rate (FAR)}

The false acceptance rate, or FAR, is the measure of the probability likelihood that the system will incorrectly accept an access attempt by an unauthorized user. A system's FAR typically is stated as the ratio of the number of false acceptances divided by the number of identification attempts.

\section{b) False Rejection Rate (FRR)}

The false rejection rate (FRR), is the measure of the likelihood that the system will incorrectly reject an access attempt by an authorized user. A system's FRR typically is stated as the ratio of the number of false rejections divided by the number of identification attempts.

\section{c) True Acceptance Rate (TAR)}

True Acceptance Rate (TAR) measures representation of the degree that the system is able to correctly match the information from the same person. Researcher of the current era is attempting to maximize this measure.

\section{d) True Rejection Rate (TRR)}

True Rejection Rate (TRR) represents the frequency of cases when information from one person is correctly not matched to any records in a database because, in fact, that person is not in 
the database. Researcher is attempting to maximize this measure.

Total accuracy is calculated by following formula:

formula: Accuracy $=100-(F A R+F R R) / 2$

The system result is compare to available statistics performance in the literature, in that the author got the highest accuracy as when FAR is 5.24 and FRR is 4.0 as show in table 3 . The results are slightly improves as compare to other results. The system performance is the compare with the other system available in literature for offline signature recognition. The proposed research attempted best accuracy as compared to reported result in literature [17-21].

\subsection{Performance of Dynamic Time Warping ( DTW )}

For checking the performance of the Dynamic Time Warping (DTW) system WER (Word Error Rate ) and accuracy.

It is a used to check the performance of the system.

It is a valuable tool for comparing different systems as well as for evaluating improvements within one system. It basically gives the errors that may have occurred in the recognition of signatures in percentage form.

Here is the formula of calculating it:

$$
\text { accuracy }=\frac{n-i}{n} * 100
$$

$\mathrm{n}=$ Total no of signatures $\mathrm{I}=$ Not Correct matched signatures

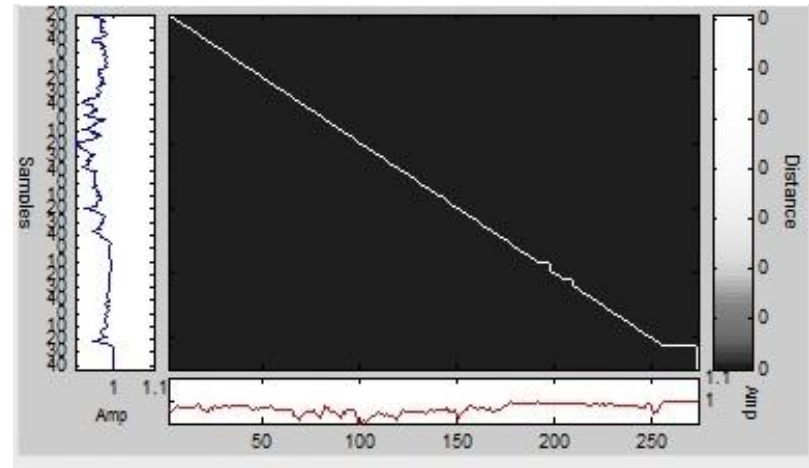

( A)

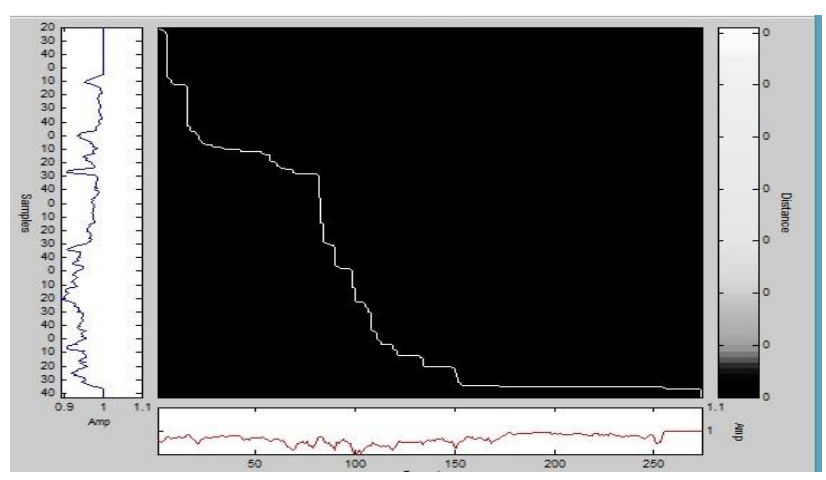

( B )

Figure 3. The graphical representation of DTW accumulates distance of two $[A]$ same signature $[B]$ Different signatures.

Table 4. The results of Word Error Rate (WER) for Horizontal projection with Vertical projection and Accuracy

\begin{tabular}{|l|l|l|l|l|}
\hline $\begin{array}{l}\text { Test } \\
\text { signature }\end{array}$ & $\begin{array}{l}\text { No. of } \\
\text { token } \\
\text { passed }\end{array}$ & $\begin{array}{l}\text { WER } \\
\text { (horizontal) }\end{array}$ & $\begin{array}{l}\text { WER } \\
\text { (vertica } \\
\text { l) }\end{array}$ & Accuracy \\
\hline Subject 1 & 10 & 40 & 60 & 50 \\
\hline Subject 2 & 10 & 90 & 60 & 80 \\
\hline Subject 3 & 10 & 70 & 80 & 75 \\
\hline Subject 4 & 10 & 70 & 90 & 80 \\
\hline Subject 5 & 10 & 70 & 80 & 25 \\
\hline Subject 6 & 10 & 80 & 80 & 80 \\
\hline Subject 7 & 10 & 70 & 100 & 85 \\
\hline Subject 8 & 10 & 100 & 80 & 75 \\
\hline Subject 9 & 10 & 20 & 0 & 10 \\
\hline Subject 10 & 10 & 90 & 80 & 85 \\
\hline
\end{tabular}

The result of comparison between the both techniques MSER and Dynamic Time Warping DTW is described in table 5.

Table 5. The comparative between Accuracy of ( MSER ) and Dynamic Time Warping ( DTW ) techniques .

\begin{tabular}{|l|l|l|}
\hline Test signature & $\begin{array}{l}\text { Accuracy of } \\
\text { MSER }\end{array}$ & $\begin{array}{l}\text { Accuracy of } \\
\text { DTW }\end{array}$ \\
\hline Subject 1 & 87.5 & 50 \\
\hline Subject 2 & 80 & 80 \\
\hline Subject 3 & 87.5 & 75 \\
\hline Subject 4 & 90 & 80 \\
\hline Subject 5 & 87.5 & 25 \\
\hline Subject 6 & 90 & 80 \\
\hline Subject 7 & 82.5 & 85 \\
\hline Subject 8 & 95 & 75 \\
\hline Subject 9 & 92.5 & 10 \\
\hline Subject 10 & 95 & 85 \\
\hline
\end{tabular}




\section{CONCLUSION}

Signature as one of the behavioural biometric is crucial for the person authentication in our daily life. This paper covered the comparisons of offline signature recognition between MSER and Dynamic Time Warping (DTW). Both of the systems are giving very positive results. MSER proved to be more efficient than DTW .

\section{REFERENCES}

[1] Deepak Tiwari and Bhawana Sharma, "Development of intelligent network for offline signature verification using pixel density, directional method and both method together" international Journal of Computer Trends and Technology- volume3Issue3- 2012, ISSN: 2231-2803, Pp403-411.

[2] Rasha Abbas, Department of Computer Science, RMIT, Master's thesis, "A prototype System for offline signature verification using multilayered feed forward neural networks", 1996

[3] Shashi Kumar D R, K B Raja, R. K Chhotaray and Sabyasachi Pattanaik "off-line Signature Verification Based on Fusion of Grid and Global Features Using Neural Networks" International Journal of Engineering Science and Technology Vol. 2(12), 2010, ISSN: 09755462, pp7035-7044.

[4] Ostu N., "A Threshold Selection Method from Gray Level Histogram", IEEE Trans. on Systems, Man and Cybernetics, SMC-8, 1978, pp. 62-66.

[5] Gonzalez R.C., Woods E., "Digital Image Processing", Addison-Wesley, 1993.

[6] Salembier, Philippe; A. Oliver as and L. Garrison (1998), "Anti-extensive Connected Operators for Image and Sequence Processing". IEEE Transactions on Image Processing 7 (4): 555-570.

[7] J. Matas, O. Chum, M. Urban and T. Pajdla. "Robust wide baseline stereo from maximally stable extremely regions." Proc. of British Machine Vision Conference, pages 384-396, 2002.

[8] K. Mikolajczyk, T. Tuytelaars, C. Schmidt, A. Fisherman, T. Kadir and L. Van Gool, "A Comparison of Affine Region Detectors"; International Journal of Computer Vision, Volume 65, Numbers 1-2 / November, 2005, pp 43-72

[9] L. Najman and M. Couprie, "Building the component tree in quasi-linear time", IEEE Transaction on Image Processing, Volume 15, Numbers 11, 2006, pp 35313539

[10] Denser, M. and Bishop, H., " Efficient Maximally Stable Extremely Region (MSER) Tracking”, CVPR, 2006.
[11] Muzaffar Bashir, Jürgen Kempf, "Reduced Dynamic Time Warping for Handwriting Recognition Based on Multi-dimensional Time Series of a Novel Pen Device", In International Journal of Intelligent Systems and Technologies, vol.3.4, Paris WASET Fall 2008.

[12] Eamonn J. Keogh, Michael J. Pazzani, "Derivative Dynamic Time Warping" In Proc. Of the 1st SIAM Int.Conf. on Data Mining (SDM-2001)

[13] Eamonn J. Keogh, Michael J. Pazzani. "Scaling up dynamic Time Warping for Data mining Applications". In Proc. 6th Int. Conf. on Knowlegde Discovery and Data Mining. KDD(2000).

[14] H. Sakoe, S. Chiba, "Dynamic programming algorithm optimization for spoken word recognition". IEEE Transaction on Acoustics, Speech and Signal Processing, Vol 26, NO1, pp. 43-49. February 1978.

[15] Hifzan Ahmed, Shailja Shukla, "Global Features based Static Signature Verification system Using DTW", International Journal of Systems , Algorithms \& Applications, vol 2, Issue 4, pp. 13-17, April 2012.

[16] V A Bharadi, H B Kekre "Off-Line Signature Recognition Systems", (C2010 International Journal of Computer Applications (0975 - 8887) Volume 1 - No. 27.

[17] S. Lee and J. C. Pan, "Off-line tracing and representation of Signatures," IEEE Trans. Syst., Man and Cyber. vol. 22, no. 4, pp. 755-771, July/August 1992.

[18] S. Chen and S. Srihari, "Use of Exterior Contours and Shape Features in Off-line Signature Verification", Proceedings of the 2005 Eight International Conference on Document Analysis and Recognition (ICDAR'05), $1520-5263 / 05$

[19] H. Batsakis, N. Papadakos, "A new signature verification technique based on a two-stage neural network classifier", Engineering Applications of Artificial Intelligence 14 (2001) 95 \pm 103 , 0952-1976/01

[20] M. K camera, S. Shorthair, "Offline Signature Verification and Identification Using Distance Statistics", International

[21] Journal of Pattern Recognition and Artificial Intelligence Vol. 18, No. 7 (2004) 1339-1360, World Scientific Publishing Company.

[22] Y. Xuhua, F. Takashi, K. Obata, Y. Uchikawa, "Constructing a High Performance Signature Verification System Using a GA Method", IEEE Conf. ANNES, 2023 Nov. 1995, PP: $170 \quad$ - 173 10.1109/ANNES.1995.499465 